\title{
Zoledronate induces autophagic cell death in human umbilical vein endothelial cells via Beclin-1 dependent pathway activation
}

\author{
YONG LU, ZHIYONG WANG, WEI HAN and HAO LI \\ Department of Oral and Maxillofacial Surgery, Nanjing Stomatological Hospital, \\ Medical School of Nanjing University, Nanjing, Jiangsu 210008, P.R. China
}

Received October 16, 2015; Accepted September 2, 2016

DOI: $10.3892 / \mathrm{mmr} .2016 .5834$

\begin{abstract}
Zoledronatehasbeenreportedtoexhibitpro-apoptotic and anti-angiogenic effects in endothelial cells, which partially contributes to bisphosphonate-associated osteonecrosis of the jaw (BP-ONJ). Zoledronate can also induce autophagic cell death. The present study hypothesized that Zoledronate may activate autophagy to exert pro-apoptotic effects in endothelial cells and aimed to investigate the effect of Zoledronate on human umbilical vein endothelial cells (HUVECs) and explore the underlying mechanisms. The current study demonstrated that Zoledronate induced autophagy in HUVECs in a dose-dependent manner, as demonstrated by increased levels of microtubule-associated proteins 1A/1B light chain 3B-II (LC3B-II) and Beclin-1, and decreased levels of sequestome 1 (SQSTM1). In addition, treatment with chloroquine further increased LC3B-II and increased SQSTM1 levels, indicating that Zoledronate induces autophagy by increasing autophagic activity. Flow cytometry and Hoechst 33258 staining revealed that inhibition of autophagy with 3-methyladenine markedly attenuated Zoledronate-induced apoptosis. Furthermore, genetic knockdown of Beclin-1 significantly inhibited autophagy and apoptosis induced by Zoledronate. The present study therefore demonstrated that Zoledronate may promote Beclin-1-mediated autophagy to induce endothelial cell apoptosis, and suggests that blocking autophagy may represent a novel approach for the prevention of BP-ONJ in patients receiving Zoledronate.
\end{abstract}

\section{Introduction}

Bisphosphonates (BPs) are pharmacological inhibitors of bone resorption that have been in use for $>30$ years and are commonly used to treat diseases associated with excessive

Correspondence to: Dr Yong Lu, Department of Oral and Maxillofacial Surgery, Nanjing Stomatological Hospital, Medical School of Nanjing University, 30 Zhongyang Road, Nanjing, Jiangsu 210008, P.R. China

E-mail: yonglu2008@yeah.net

Key words: zoledronate, autophagy, apoptosis, Beclin-1 bone loss, including osteoporosis, multiple myeloma and complications associated with bone cancer metastases (1-3).

Zoledronate is a third-generation BP, approved for the treatment of cancer-induced bone diseases, with potent therapeutic effects in suppressing osteoclastic activity and resorptive bone loss $(4,5)$. However, long-term use of Zoledronate can result in BP-associated osteonecrosis of the jaw (BP-ONJ) (6-8). BP-ONJ is most commonly associated with patients with malignancies that receive high-dose intravenous BP, occurring significantly less frequently in patients with osteoporosis that receive orally-administered BP (9). Previous studies have demonstrated that Zoledronate can promote osteoclast apoptosis by inhibiting enzymes of the mevalonate pathways (10). However, in addition to osteoclast inhibition, osteoblasts and osteocytes are also inhibited by Zoledronate, leading to suppression of bone remodeling $(11,12)$. Additionally, BP-ONJ has been associated with BP-induced anti-angiogenic effects; failed wound healing is occasionally observed following protracted Zoledronate use, which may result in secondary necrotic bone $(13,14)$. Clinical and experimental studies have also indicated that Zoledronate induces endothelial cell apoptosis and subsequently reduces angiogenesis $(13,15,16)$. Notably, Zoledronate can reduce the number of endothelial cells within alveolar bone, particularly following tooth extraction, due to greater accumulation of Zoledronate here than in other sites of the skeleton $(11,17)$. However, the regulatory mechanism by which Zoledronate promotes endothelial cell apoptosis has not been clearly elucidated at the molecular level.

Autophagy is a highly regulated process, involved in the degradation and recycling of proteins, intracellular pathogens and cytoplasmic organelles (18) and is important for cell survival, differentiation and homeostasis $(19,20)$. Previous studies have demonstrated Zoledronate-induced autophagy in various tumor cells $(18,21-23)$. However, the ability of Zoledronate to induce autophagy in endothelial cells remains unknown. In addition, autophagy has been suggested to be involved in crosstalk with apoptosis by inhibiting or promoting the process $(21,24)$. Thus, the present study hypothesized that Zoledronate may induce apoptosis in human umbilical vein endothelial cells (HUVECs), partially, by affecting autophagy. In addition to confirmation of Zoledronate-induced apoptosis in HUVECs, the present study also aimed to provide evidence of the critical function of autophagy in Zoledronate-induced apoptosis. 


\section{Materials and methods}

Reagents. Endothelial basal medium (EBM), penicillin, streptomycin, fetal calf serum (FCS), human endothelial growth factor $\beta$ ( $\beta$-ECGF), TRIzol reagent and Lipofectamine 2000 were purchased from Invitrogen (Thermo Fisher Scientific, Inc., Waltham, MA, USA). Zoledronate (full name, 2-(imidazole-1-yl)-hydroxy-ethylidene-1, 1-bisphosphonic acid, disodium salt, 4.75 hydrate; molecular weight, 401.6) was purchased from Novartis International AG (Basel, Switzerland). A stock solution of Zoledronate $(100 \mathrm{mM})$ was prepared in filter-sterilized phosphate-buffered saline (PBS). Chloroquine, 3-methyladenine (3-MA), 3-(4,5-dimethylthiazol-2-yl)-2,5-diphenyltetrazolium bromide (MTT) and Hoechst 33258 were obtained from Sigma-Aldrich (Merck Millipore, Darmstadt, Germany).

Cell culture. Human umbilical cord veins were obtained from the Affiliated Nanjing University Medical School and human umbilical vein endothelial cells (HUVECs) were isolated as previously described (25). The study was approved by the Medical Research Ethics Committee of Medical School of Nanjing University and informed content was obtained from 20 pregnant women giving birth between 2012 and 2014. In brief, cells were harvested from umbilical cord by $0.125 \%$ trypsin, and then cultured in EBM supplemented with $100 \mathrm{U} / \mathrm{ml}$ penicillin, $100 \mu \mathrm{g} / \mathrm{ml}$ streptomycin, 20\% FCS and $100 \mu \mathrm{g} / \mathrm{ml}$ EGF in an incubator with $5 \% \mathrm{CO}_{2}$ and $95 \%$ air at $37^{\circ} \mathrm{C}$.

Reverse transcription-quantitative polymerase chain reaction (RT-qPCR) for microtubule-associated proteins $1 A / 1 B$ light chain $3 B(L C 3 B)$ expression. Total RNA from HUVECs treated with various concentrations of Zoledronate $(25,50$, 75 and $100 \mu \mathrm{M}$ ) for $48 \mathrm{~h}$ was isolated using TRIzol reagent according to the manufacturer's protocols. Total RNA $(1 \mu \mathrm{g})$ was reverse-transcribed using SuperScript III First-Strand Synthesis system (Invitrogen; Thermo Fisher Scientific, Inc.) according to the manufacturer's instructions. Amplification was performed using Fast SYBR ${ }^{\circledR}$ Green Master Mix Kit (Applied Biosystems; Thermo Fisher Scientific, Inc.) in an Applied Biosystems 7300 Fast RT-PCR system and LC3B expression was calculated using the $2^{-\Delta \Delta \mathrm{Cq}}$ method as previously described (26). A total of 35 cycles were conducted consisting of denaturation at $95^{\circ} \mathrm{C}$ for $15 \mathrm{sec}$, primer annealing at $60^{\circ} \mathrm{C}$ for $1 \mathrm{~min}$ and primer extension at $72^{\circ} \mathrm{C}$ for $30 \mathrm{sec}$. The specific primer sequences used in this study were as follows: LC3B, forward, 5'-AGCAGCATCCAACCA AAATC-3', reverse, 5'-CTGTGTCCGTTCACCAACAG-3'; 18S, forward, 5'-CGGCTACCACATCCAAGGAA-3', reverse, 5'-CTGGAA TTACCGCGGCT-3'.

Western blot analysis. Proteins from HUVECs $\left(2 \times 10^{5}\right.$ cells $)$ treated with various concentrations of Zoledronate $(25,50,75$ and $100 \mu \mathrm{M}$ ) for $48 \mathrm{~h}$ were isolated with radioimmunoprecipitation assay buffer (Beyotime Institute of Biotechnology, Jiangsu, China) containing protease and phosphatase inhibitor cocktail (Merck Millipore, Darmstadt, Germany). The protein content of cell lysates was determined by Bradford assay (Bio-Rad Laboratories, Inc., Hercules, CA, USA). An equal amount of protein $(50 \mu \mathrm{g})$ from HUVEC lysates was separated by $10-12 \%$ sodium dodecyl sulfate-polyacrylamide gel electrophoresis, blotted onto Immobilon-P membranes (Merck Millipore, Volketswil, Switzerland), blocked with 5\% non-fat milk with TBST (Tris-HCL $20 \mathrm{mM}, \mathrm{NaCl} 150 \mathrm{mM}$, $0.1 \%$ Tween 20) and then incubated with antibodies against LC3B (cat. no. 3868), sequestome 1 (SQSTM1) (cat. no. 8025), Beclin-1 (cat. no. 3738), cleaved caspase-9 (cat. no. 7237), cleaved caspase-3 (cat. no. 9579) and $\beta$-actin (cat. no. 3700) (1:1,000; Cell Signaling Technology, Inc., Danvers, MA, USA) overnight at $4^{\circ} \mathrm{C}$. Following incubation with the horseradish peroxide-labeled goat anti-rabbit (cat. no. A0208) or goat anti-mouse (cat. no. A0216) antibody (Beyotime Institute of Biotechnology) at room temperature for $1 \mathrm{~h}$, the signals were visualized on radiographic film using enhanced chemiluminescence reagents (Bio-Rad Laboratories, Inc.). The density of the appropriate sized bands was quantified using ImageJ software, version 1.41 (National Institutes of Health, Bethesda, MD, USA).

Green fluorescent protein (GFP)-LC3 adenovirus infection and assay. Adenovirus encoding GFP-LC3 (Ad-GFP-LC3) was obtained from Cyagen Biotechnology Co., Ltd. (Santa Clara, CA, USA). HUVECs $\left(2 \times 10^{5}\right.$ cells) were cultured in EBM containing 2\% FCS and Ad-GFP-LC3 (multiplicity of infection, 100) for $3 \mathrm{~h}$, following which, the medium was replaced with fresh complete medium. HUVECs were infected with Ad-GFP-LC3 for $24 \mathrm{~h}$ prior to treatment with various concentrations of Zoledronate for a further $48 \mathrm{~h}$. Following treatment, cells were harvested and fixed in $4 \%$ paraformaldehyde and visualized and photographed in Zeiss LSM 5 Pascal laser scanning confocal fluorescent microscope (Zeiss AG, Oberkochen, Germany). The average number of GFP-LC3 punctae per cell were counted to quantify autophagy activities using ImageJ software, version 1.41 (National Institutes of Health).

Cell viability assay. Cell viability was determined by MTT assay. HUVECs $\left(2 \times 10^{3}\right.$ cells/well) were plated in 96-well culture plates and rendered quiescent by culturing in serum-free medium overnight at $37^{\circ} \mathrm{C}$. Following treatment with different concentrations of Zoledronate for $48 \mathrm{~h}, 10 \mu \mathrm{l}$ MTT $(5 \mathrm{mg} / \mathrm{ml})$ was added to each well, and plates incubated for a further $4 \mathrm{~h}$. The medium was removed and $100 \mu 1$ of dimethyl sulfoxide was added to solubilize the formazan crystals. Absorbance of the medium was measured at $570 \mathrm{~nm}$ using the SPECTRAMax M5 reader (Molecular Devices, LLC, Sunnyvale, CA, USA).

Cell apoptosis assay. HUVEC were treated with various concentrations of Zoledronate (25, 50, 75 and $100 \mu \mathrm{M})$ for another $48 \mathrm{~h}$, or pretreated with 3-MA $(5 \mathrm{mM})$ for $30 \mathrm{~min}$ prior to Zoledronate $(100 \mu \mathrm{M})$ incubation for another $48 \mathrm{~h}$. Cell apoptosis was evaluated by fluorescein isothiocyanate (FITC)-conjugated Annexin-V and propidium iodide (PI) staining by using FITC-Annexin V and PI double staining kit (KeyGen Biotech Co., Ltd, Nanjing, China) according to the manufacturer's protocols. Treated or untreated cells $\left(2 \times 10^{5}\right)$ were digested and suspended in $500 \mu \mathrm{l}$ binding buffer plus $5 \mu \mathrm{l}$ FITC-labeled Annexin V and $5 \mu \mathrm{l}$ PI solution. The mixtures were incubated on ice for $10 \mathrm{~min}$, and then plotted for FITC-conjugated Annexin V and PI in a two-way dot plot to 
count the apoptotic cells with flow cytometry (BD Biosciences, San Jose, CA, USA).

Hoechst 33258 dye staining. Apoptotic morphology of HUVECs was observed by Hoechst 33258 dye. Cells were washed twice with PBS, fixed in $4 \%$ paraformaldehyde for $10 \mathrm{~min}$, permeabilized with $0.1 \%$ Triton X-100 and incubated with $2.5 \mu \mathrm{g} / \mu \mathrm{l}$ Hoechst 33258 for $5 \mathrm{~min}$ at room temperature. Stained cells were washed, and nuclei observed by fluorescence microscopy using an IX83 inverted motorized microscope (Olympus Corporation, Tokyo, Japan).

Small interfering RNA (siRNA) transfection. Beclin-1 siRNA (5'-GGUCUAAGACGUCCAACAA-3') and non-targeting negative control siRNA (5'-UGGUUUACAUGUCGA CUAA-3'; Invitrogen; Thermo Fisher Scientific, Inc.) were transiently transfected with Lipofectamine 2000 (Invitrogen; Thermo Fisher Scientific, Inc.) according to the manufacturer's protocol. Briefly, siRNA and Lipofectamine 2000 were mixed at ratio of $1: 4$, then incubated in serum- and antibiotic-free EBM for $15 \mathrm{~min}$. The mixture was added to $70 \%$ confluence of HUVECs and swirled gently to ensure uniform distribution. Following $3 \mathrm{~h}$ incubation at room temperature, the transfection mixture was removed and fresh complete medium was added prior to further incubation. HUVECs were incubated for $24 \mathrm{~h}$ following siRNA transfection, followed by $48 \mathrm{~h}$ Zoledronate $(100 \mu \mathrm{M})$ treatment.

Statistical analysis. All data are expressed as the mean \pm standard error, and $\mathrm{n}$ values indicate the number of independent experiments performed. Comparisons between multiple groups were analyzed by one-way analysis of variance, followed by Tukey's multiple comparison post-hoc test. All statistical analyses were performed using SPSS software (version 17.0; SPSS Inc., Chicago, IL, USA). P $<0.05$ was considered to indicate a statistically significant difference.

\section{Results}

Zoledronate induces the expression of markers for autophagy in HUVECs. qPCR analysis of HUVECs revealed a significant dose-dependent increase in the mRNA expression levels of LC3B following Zoledronate treatment (Fig. 1A). To further confirm autophagy in HUVECs was mediated by Zoledronate, the conversion of LC3B-I to LC3B-II was analyzed, as this suggests an increase in the number of autophagosomes within cells (27). Although the protein level of LC3B-I was marginally increased, Zoledronate treatment significantly increased the levels of LC3B-II and the ratio of LC3B-II/I compared with untreated cells $(\mathrm{P}<0.05$; Fig. 1B). Furthermore, infection with GFP-LC3 adenovirus revealed a dose-dependent increase in the number of GFP puncta in the cytoplasm following Zoledronate exposure, indicating increased transformation of LC3B-I to LC3B-II ( $<<0.05$; Fig. $1 \mathrm{C}$ and D). By contrast, the levels of SQSTM1, which is degraded in the lysosome following autophagosome fusion (28), were significantly decreased in HUVECs that were treated with $>50 \mu \mathrm{M}$ Zoledronate in a dose-dependent manner $(\mathrm{P}<0.01$; Fig. 1E). Western blot analysis of another autophagy marker, Beclin-1, which is required for the formation of autophagosomes (29), revealed that the protein expression level of Beclin-1 was increased dose-dependently following exposure to Zoledronate $(\mathrm{P}<0.05$; Fig. 1F). Zoledronate was, therefore, demonstrated to induce autophagy in HUVECs.

Zoledronate induces autophagic flux in HUVECs. Increased autophagy can be attributed either to enhanced autophagosome formation or reduction of lysosomal activity (30). Since the increased autophagosome formation or the decreased lysosomal degradation may result in LC3B-II accumulation, the protein expression levels of LC3B-II were measured in HUVECs treated with $100 \mu \mathrm{M}$ Zoledronate in the presence or absence of the lysosome inhibitor, chloroquine $(1 \mu \mathrm{M})$. Western blot analysis revealed that pretreatment with chloroquine increased basal LC3B-II protein expression levels compared with vehicle-treated cells $(\mathrm{P}<0.01)$ and further enhanced the Zoledronate-induced increase in LC3B-II levels compared with cells treated with Zoledronate only $(\mathrm{P}<0.01$; Fig. 2A). Furthermore, accumulation of SQSTM1 was also enhanced in HUVECs following inhibition of lysosomal activity by chloroquine compared with vehicle-treated cells $(\mathrm{P}<0.01)$, and chloroquine treatment abolished the inhibitory effect of Zoledronate on SQSTM1 levels ( $\mathrm{P}<0.01$; Fig. 2B). Zoledronate was therefore demonstrated to induce autophagy through increased autophagic activity rather than inhibition of lysosome degradation.

Inhibition of autophagy attenuates Zoledronate-induced apoptosis in HUVECs. The effect of Zoledronate on the viability or apoptosis of HUVECs was investigated, due to the intricate crosstalk between autophagy and apoptosis (24). Zoledronate was demonstrated to significantly decrease the viability of HUVECs in a dose-dependent manner compared with untreated control $(\mathrm{P}<0.01)$, as demonstrated in Fig. 3A. Cell viability following treatment with $100 \mu \mathrm{M}$ Zoledronate was decreased to $68.4 \pm 5.8 \%$ compared with untreated control cells $(\mathrm{P}<0.01$; Fig $3 \mathrm{~A})$.

To further determine whether Zoledronate-induced cell death was due to apoptosis, molecular and cellular changes associated with apoptosis were analyzed. Increased activation of caspase signaling was observed, as demonstrated by a significant increase in the levels of cleaved caspase- 9 and caspase- 3 following Zoledronate treatment, compared with untreated cells $(\mathrm{P}<0.05$; Fig. $3 \mathrm{~B}$ and $\mathrm{C})$. HUVECs were subsequently stained with FITC-Annexin-V to detect early apoptotic cells, and PI to detect late apoptotic/necrotic cells (31). Dose-dependently increased PI and Annexin-V cell staining was observed in Zoledronate-treated cells compared with untreated control cells (Fig. 3D), resulting in a significant dose-dependent increase in the total apoptosis rate $(\mathrm{P}<0.01$; Fig. 3E). However, the increased apoptosis induced by $100 \mu \mathrm{M}$ Zoledronate was significantly inhibited by $59.2 \pm 6.7 \%$ following pretreatment with $5 \mathrm{mM} 3-\mathrm{MA}$, an autophagy inhibitor $(\mathrm{P}<0.01$; Fig. 3D and $\mathrm{E})$. 3-MA treatment alone exerted no effect on cell apoptosis (data not shown). Increased nuclear fragmentation in Zoledronate-treated HUVECs compared with untreated control cells was also observed by Hoechst 33258 staining ( $\mathrm{P}<0.01$; Fig. $3 \mathrm{~F}$ and $\mathrm{G})$, and was attenuated by inhibition of autophagy with 3-MA $(\mathrm{P}<0.01$; Fig. 3F and G). Increased autophagy is, therefore, 
A

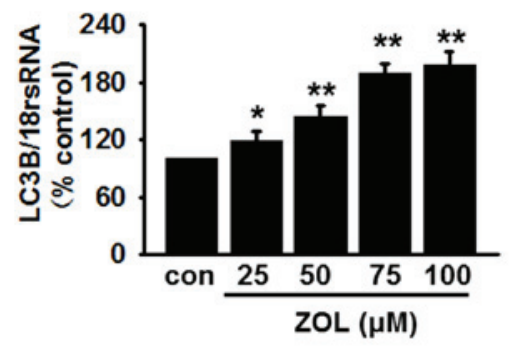

$\mathrm{C}$

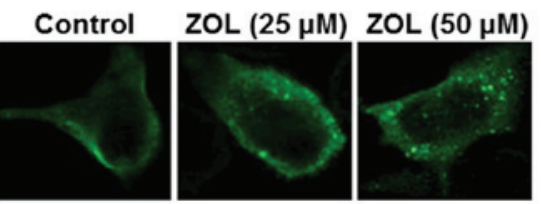

ZOL $(75 \mu \mathrm{M}) \mathrm{ZOL}(100 \mu \mathrm{M})$
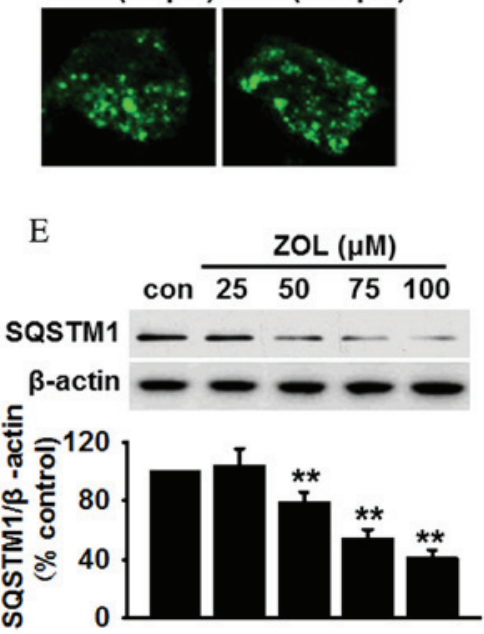

B

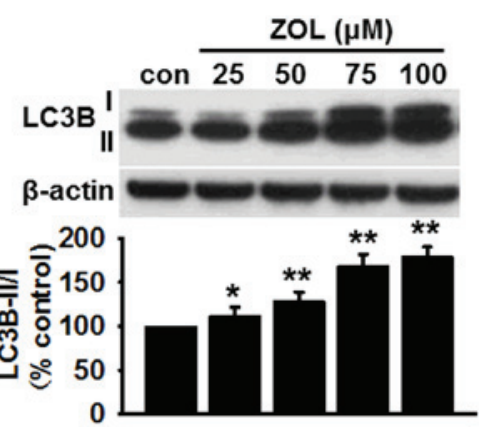

$\mathrm{D}$

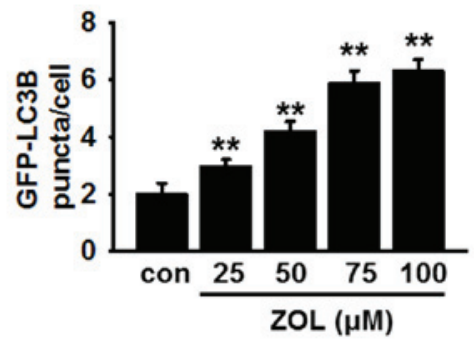

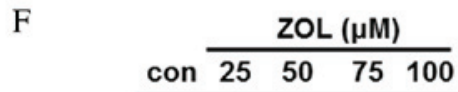
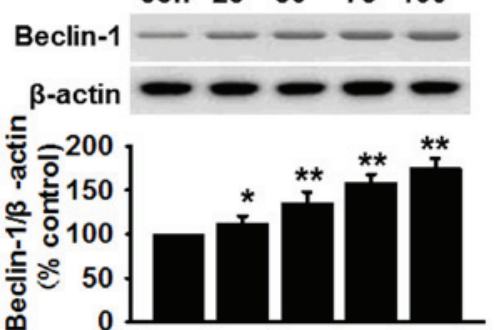

Figure 1. ZOL induces dose dependent autophagy in HUVECs. (A) Cells were treated with 25, 50, 75 and $100 \mu \mathrm{M}$ ZOL for $48 \mathrm{~h}$, then the mRNA expression levels of LC3B were determined by reverse transcription-quantitative polymerase chain reaction. (B) The levels of LC3B-I and LC3B-II were analyzed by semi-quantitative western blot. (C) HUVECs were infected with Ad-GFP-LC3 adenovirus prior to ZOL treatment, following which, GFP-LC3 punctae were observed using a laser scanning confocal fluorescent microscope. A representative single cell exhibits LC3 punctae as a marker of autophagic vesicles. (D) Quantification of the mean number of GFP-LC3 punctae per cell. Representative blots and quantitative bar graphs demonstrating the expression of (E) SQSTM1 and (F) Beclin-1, following ZOL treatment. All data are presented as the mean \pm standard error. ${ }^{*} \mathrm{P}<0.05,{ }^{* *} \mathrm{P}<0.01 \mathrm{vs}$. untreated control, $\mathrm{n}=4-6$. HUVECs, human umbilical vein endothelial cells; LC3B, microtubule-associated proteins 1A/1B light chain 3B; 18S rRNA, 18S ribosomal RNA; Con, control; ZOL, Zoledronate; SQSTM1, sequestome 1.
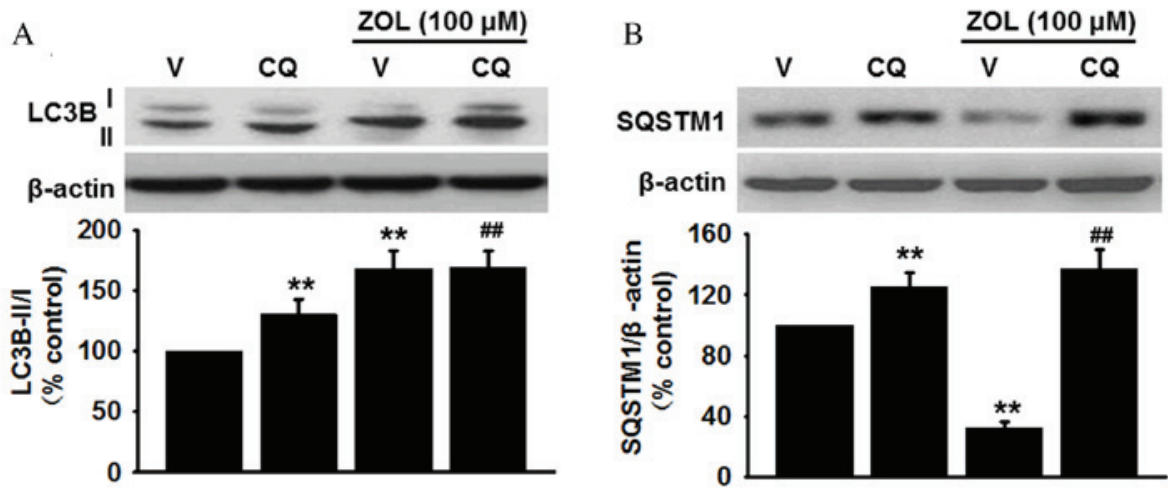

Figure 2. ZOL increases autophagic activity instead of inhibition of lysosomal degradation. Human umbilical vein endothelial cells were pretreated with vehicle or $1 \mu \mathrm{M} \mathrm{CQ}$ for $1 \mathrm{~h}$, then exposed to $100 \mu \mathrm{M}$ ZOL for $48 \mathrm{~h}$. The protein levels of (A) LC3B-I and -II, and (B) SQSTM1 were determined by western blot and densitometric analysis was performed. Data are presented as the mean \pm standard error, $\mathrm{n}=6 .{ }^{* *} \mathrm{P}<0.01 \mathrm{vs}$. V alone; ${ }^{\# \#} \mathrm{P}<0.01 \mathrm{vs}$. ZOL $+\mathrm{V}$ group; $\mathrm{ZOL}$, Zoledronate; V, vehicle; CQ, chloroquine; LC3B, microtubule-associated proteins 1A/1B light chain 3B; SQSTM1, sequestome 1. 
A

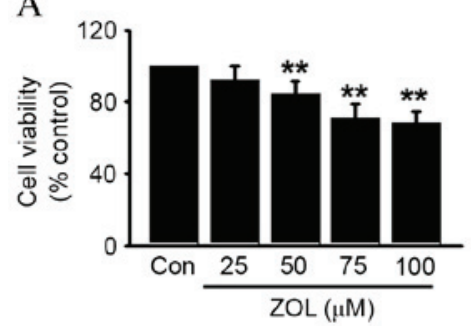

B

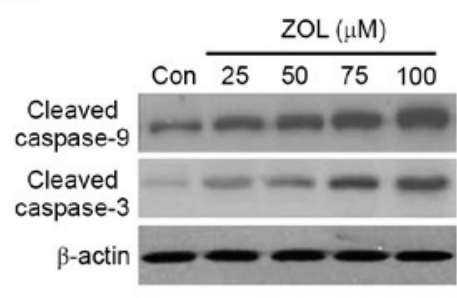

C

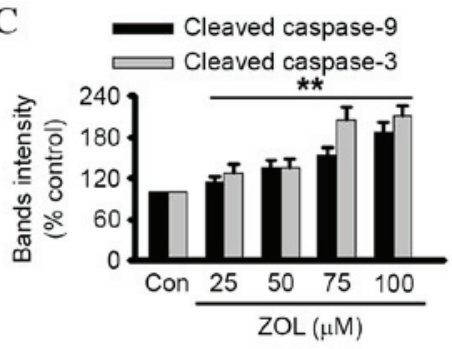

D

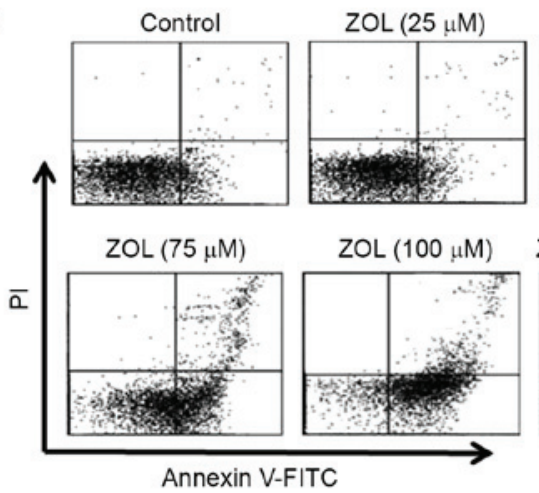

F

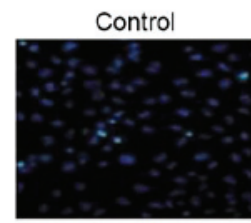

ZOL

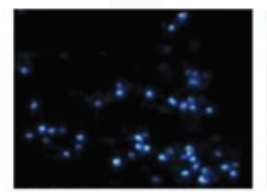

3-MA

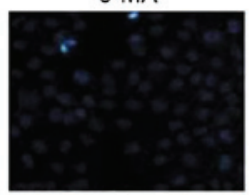

ZOL+3-MA

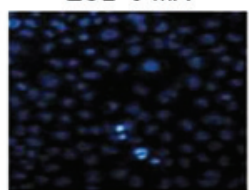

E
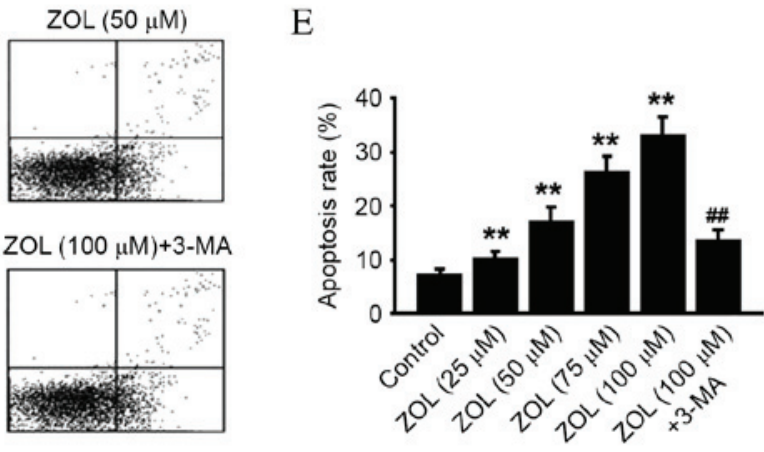

G

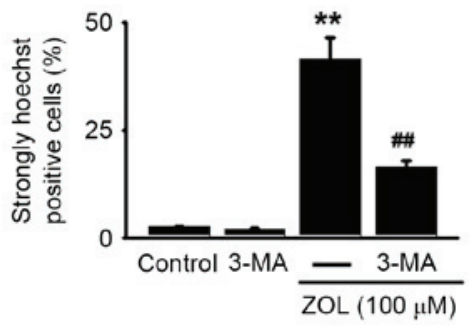

Figure 3. Inhibition of autophagy protects against ZOL-induced HUVEC apoptosis. HUVECs were incubated with various concentrations of ZOL for $48 \mathrm{~h}$, then (A) cell viability was assessed by 3-(4,5-dimethylthiazol-2-yl)-2,5-diphenyltetrazolium bromide (MTT) assay and (B) cleaved caspase-9 and -3 were detected by western blot. Representative western blot images are presented. (C) Densitometric analysis of caspase-9 and cleaved caspase-3 western blots was performed. HUVECs were treated with $5 \mathrm{mM} 3$-MA prior to ZOL treatment, then (D) the number of apoptotic cells were determined by Annexin V-FITC/PI staining using flow cytometry, (E) followed by quantitative analysis of the percentage of apoptotic cells. HUVECs treated with $5 \mathrm{mM} 3-\mathrm{MA}$ and/or $100 \mu \mathrm{M}$ ZOL were fixed and their DNA stained with Hoechst 33258. (F) Confocal images of nuclear staining are presented and (G) quantification of the number of strongly Hoechst 33258 positive cells was performed. Data are presented as the mean \pm standard error, $\mathrm{n}=6 .{ }^{* *} \mathrm{P}<0.01$ vs. Con; ${ }^{\# \#} \mathrm{P}<0.01$ vs. $100 \mu \mathrm{M} Z \mathrm{ZOL}$. HUVECs, human umbilical vein endothelial cells; ZOL, Zoledronate; Con, untreated control; PI, propidium iodide; FITC, fluorescein isothiocyanate; 3-MA, 3-methyladenine.

indicated to be involved in Zoledronate-induced apoptosis in HUVECs.

Blockade of Beclin-1 inhibits Zoledronate-induced autophagic death in HUVECs. As Zoledronate was demonstrated to induce an increase in Beclin-1 expression, it was speculated that the autophagy induced by Zoledronate was dependent on the Beclin-1 pathway. To examine this hypothesis, Beclin-1 expression was genetically knocked down in HUVECs prior to Zoledronate exposure, and changes in cell apoptosis were measured. Transfection efficiency was confirmed by a significant decrease in Beclin-1 protein expression in siRNA-treated cells $(\mathrm{P}<0.01$; Fig. 4A). Inhibition of Beclin-1 expression decreased the basal LC3B-II protein level compared with untransfected cells $(\mathrm{P}<0.05$; Fig. 4B), and also abrogated the Zoledronate-induced increase in LC3B-II protein expression levels when compared with untransfected cells treated with
$100 \mu \mathrm{M}$ Zoledronate $(\mathrm{P}<0.01$; Fig. 4B). By contrast, inhibition of Beclin-1 expression increased basal SQSTM1 protein expression levels compared with untransfected cells $(\mathrm{P}<0.01$; Fig. 4C), and the decreased SQSTM1 protein expression levels induced by Zoledronate treatment $(\mathrm{P}<0.01$; Fig. 4C), were partially restored in Beclin-1 siRNA-transfected cells $(\mathrm{P}<0.01$; Fig. 4C).

The involvement of Beclin-1-mediated autophagy in HUVEC apoptosis following Zoledronate exposure was subsequently investigated. Beclin-1 knockdown was revealed to significantly increase cell viability in HUVECs following $48 \mathrm{~h}$ exposure to $100 \mu \mathrm{M}$ Zoledronate by MTT assay $(\mathrm{P}<0.01$; Fig. 4D) and Hoechst 33258 staining $(\mathrm{P}<0.01$; Fig. 4E and $\mathrm{F}$ ) compared with untransfected/Zoledronate-treated control cells. However, knockdown of Beclin-1 did not significantly affect HUVEC cell viability or Hoechst staining in the absence of Zoledronate compared with untransfected control 

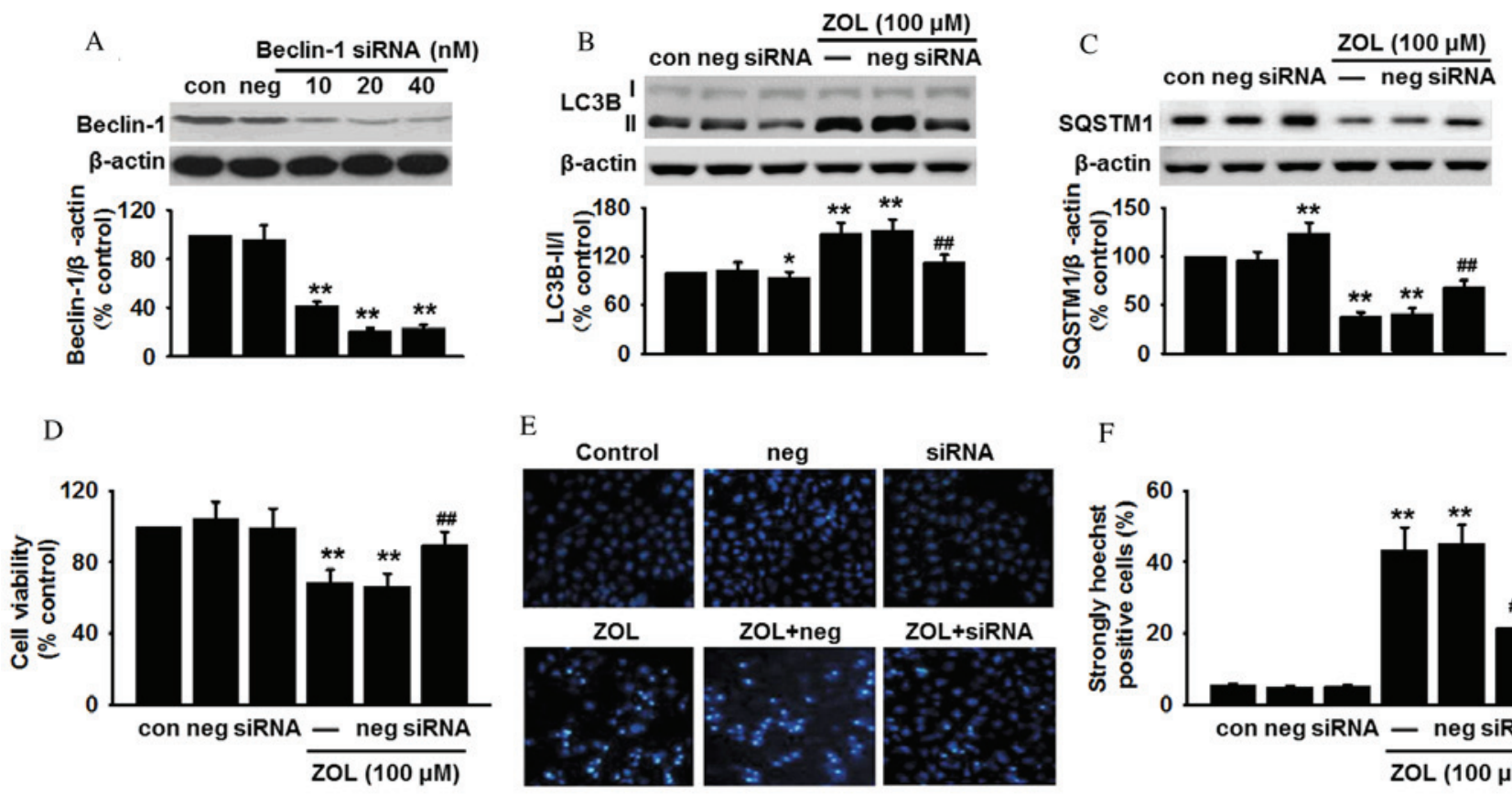

E
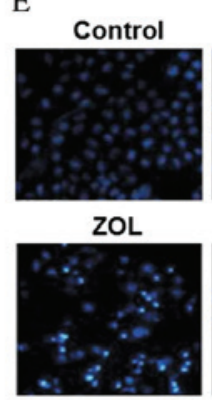

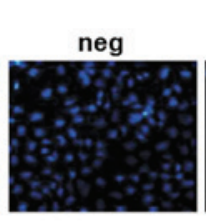

ZOL+neg

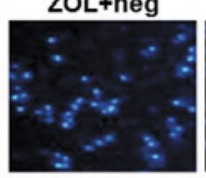

SIRNA

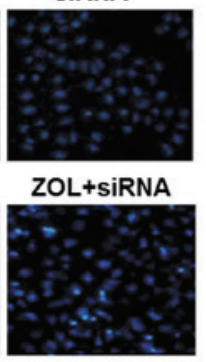

F

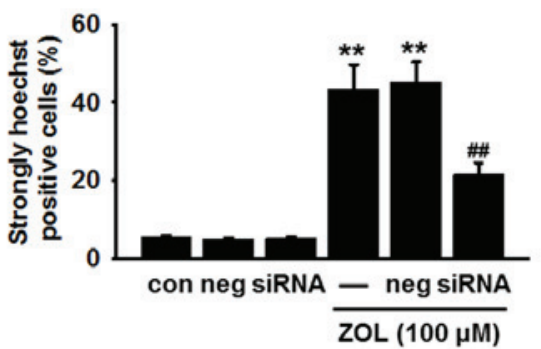

Figure 4. Absence of Beclin-1 inhibits ZOL-induced autophagic cell death. (A) HUVECs were transfected with Beclin-1 siRNA or non-targeting negative control siRNA for $24 \mathrm{~h}$, and the protein expression level of Beclin-1 determined by western blot. HUVECs were transfected with Beclin-1 siRNA or non-targeting negative control siRNA for $24 \mathrm{~h}$, followed by $48 \mathrm{~h}$ treatment with $100 \mu \mathrm{M}$ ZOL. The protein expression levels of (B) LC3B-II and (C) SQSTM1 were analyzed by western blot, (D) cell viability examined by 3-(4,5-dimethylthiazol-2-yl)-2,5-diphenyltetrazolium bromide (MTT) assay, and (E) apoptosis detected by Hoechst 33258 staining and $(\mathrm{F})$ the number of apoptotic cells quantified. Data are presented as the mean \pm standard error, $\mathrm{n}=6 .{ }^{*} \mathrm{P}<0.05,{ }^{* *} \mathrm{P}<0.01$ vs. untransfected control; ${ }^{\#} \mathrm{P}<0.01$ vs. untransfected cells treated with $100 \mu \mathrm{M} Z \mathrm{ZL}$. HUVECs, human umbilical vein endothelial cells; siRNA, small interfering RNA; Con, untransfected control; Neg, negative control siRNA; ZOL, Zoledronate; LC3B, microtubule-associated proteins 1A/1B light chain 3B; SQSTM1, sequestome 1 .

cells (Fig. 4D-F). Beclin-1 is, therefore, suggested to be important for Zoledronate-induced HUVEC apoptosis.

\section{Discussion}

Although numerous studies have suggested that long-term use of BPs contributes to the development of BP-ONJ $(1,8,11-13)$, the underlying molecular mechanism remains poorly understood. To the best of our knowledge, the present study is the first to demonstrate the ability of Zoledronate to increase autophagic flux, thus inducing autophagy in HUVECs. Additionally, inhibition of autophagy and/or Beclin-1 was demonstrated to reverse the increase in apoptosis in HUVECs treated with Zoledronate. These findings suggest a novel mechanism of endothelial cell injury induced by Zoledronate and provide a potential therapeutic approach for the treatment of BP-ONJ.

Autophagy is regulated by $>30$ autophagy specific genes, including LC3 [a mammalian homologue of autophagy-related protein 8 (Atg8)], SQSTM1 (also termed p62) and Beclin-1 (a mammalian homologue of Atg6). During autophagosome formation, LC3B-I is conjugated with phosphatidylethanolamine at its C-terminus to form LC3B-II, located in the autophagosome membrane, which then binds to SQSTM1 to facilitate autophagic degradation in the lysosome $(32,33)$. LC3B-II, SQSTM1 and Beclin-1 are, therefore, frequently used as markers of autophagy. The present study revealed that Zoledronate dose-dependently increased the expression levels of LC3B-II and Beclin-1, while decreasing the expression levels of SQSTM1, indicating induction of autophagy. This supports the previous findings that Zoledronate induced autophagy in human breast cancer cells $(18,34)$, cervical cancer cells (21), salivary adenoid cystic carcinoma cells (22) and human prostate cancer cells (23).

LC3B-II is localized inside and outside the membrane of autophagolysosomes (18). Degradation of the inner LC3B-II in the autophagolysosome can be prevented by inhibition of autophagolysosome fusion (27). Therefore, activation of autophagy may not be directly associated with enhanced autophagic activity, and may be a reduction of autophagolysosome degradation, leading to accumulation of LC3B-II or feedback stimulation of Beclin-1 (35). By inhibiting lysosomal activity with chloroquine, the current study demonstrated that Zoledronate further enhanced the expression of LC3B-II and SQSTM1 in HUVECs with reduced lysosomal activity, indicating that Zoledronate acts via increased autophagic activity rather than by reducing autophagolysosome degradation.

Neovascularization at the alveolar ridge following dental extraction requires endothelial cells during angiogenesis and vasculogenesis (17). The majority of clinicians are in agreement that tooth extraction should be avoided in patients receiving BPs and that BP treatment should not be resumed until the wound is healed completely (36). However, clinicians should be also aware that BPs can accumulate in the bone microenvironment and that the half-life of BPs can be $>10$ years (37). Therefore, it is necessary to further examine the risk factors associated with these drugs. Previous studies have demonstrated that Zoledronate inhibits the proliferation, migration and adhesion of endothelial cells, indicating that Zoledronate may attenuate the viability of endothelial cells $(16,38)$. The present study 
demonstrated that Zoledronate attenuated cell viability and induced apoptosis in HUVECs, in accordance with a previous study, which reported, that Zoledronate increased endothelial cell apoptosis and subsequently reduced angiogenesis in an experimental cervical cancer model (15).

Autophagy is a complex process, and has a dual function in apoptosis regulation, depending on the severity and type of stimuli that induces the autophagy $(27,39)$. The present study demonstrated that Zoledronate-induced apoptosis was significantly attenuated following inhibition of autophagy using 3-MA, indicating that Zoledronate increases endothelial cell apoptosis, at least partially, by inducing autophagy. Beclin-1 is important in the regulation of autophagy and the coupling of autophagy to apoptosis (29). Previous studies have demonstrated that overexpression of Beclin-1 induces autophagy and inhibits tumor development $(19,40)$. In the current study, Beclin-1 silencing resulted in inhibition of HUVEC apoptosis, suggesting that Beclin-1 suppression is important for the pro-apoptotic effect of Zoledronate.

Notably, the in vitro data in the current study is not fully representative of the processes in the body, therefore, further studies are required to verify these effects in vivo. Furthermore, the mechanisms by which Zoledronate induces autophagy in endothelial cells, for example the mevalonate pathway and oxidative stress, should be further investigated.

In summary, the present study demonstrates that Zoledronate induces HUVEC apoptosis via activation of autophagy, and that Zoledronate-induced autophagy and apoptosis is inhibited by siRNA suppression of Beclin-1. These results confirm and enhance previous findings of the impact of Zoledronate on endothelial cells, and may guide novel therapeutic strategies to prevent BP-ONJ.

\section{Acknowledgements}

This study was supported by the Nanjing Medical Science and Technique Development Foundation (grant no. QRX11125) and the Jiangsu Provincial Clinical Medicine of Science and Technology project (grant nos. BL2012017 and BL2013005).

\section{References}

1. Bassett CA, Donath A, Macagno F, Preisig R, Fleisch H and Francis MD: Diphosphonates in the treatment of myositis ossificans. Lancet 2: 845, 1969.

2. Selander KS, Mönkkönen J, Karhukorpi EK, Härkönen P, Hannuniemi R and Väänänen HK: Characteristics of clodronate-induced apoptosis in osteoclasts and macrophages. Mol Pharmacol 50: 1127-1138, 1996.

3. Brown DL and Robbins R: Developments in the therapeutic applications of bisphosphonates. J Clin Pharmacol 39: 651-660, 1999.

4. Tsai SH,Huang PH, Chang WC, Tsai HY, Lin CP, Leu HB, Wu TC, Chen JW and Lin SJ: Zoledronate inhibits ischemia-induced neovascularization by impairing the mobilization and function of endothelial progenitor cells. PloS One 7: e41065, 2012.

5. Ottewell PD, Woodward JK, Lefley DV, Evans CA, Coleman RE and Holen I: Anticancer mechanisms of doxorubicin and zoledronic acid in breast cancer tumor growth in bone. Mol Cancer Ther 8: 2821-2832, 2009.

6. Kobayashi Y, Hiraga T, Ueda A, Wang L, Matsumoto-Nakano M, Hata K, Yatani $\mathrm{H}$ and Yoneda T: Zoledronic acid delays wound healing of the tooth extraction socket, inhibits oral epithelial cell migration, and promotes proliferation and adhesion to hydroxyapatite of oral bacteria, without causing osteonecrosis of the jaw, in mice. J Bone Miner Metab 28: 165-175, 2010.
7. Yamashita J, Koi K, Yang DY and McCauley LK: Effect of zoledronate on oral wound healing in rats. Clin Cancer Res 17: 1405-1414, 2011.

8. Diel IJ, Bergner R and Grötz KA: Adverse effects of bisphosphonates: Current issues. J Support Oncol 5: 475-482, 2007.

9. Ruggiero SL, Dodson TB, Assael LA, Landesberg R, Marx RE and Mehrotra B; American Association of Oral and Maxillofacial Surgeons: American Association of Oral and Maxillofacial Surgeons position paper on bisphosphonate-related osteonecrosis of the jaws-2009 update. J Oral Maxillofac Surg 67 (5 Suppl): $2-12,2009$.

10. Russell RG: Bisphosphonates: From bench to bedside. Ann NY Acad Sci 1068: 367-401, 2006.

11. Allen MR and Burr DB: Mandible matrix necrosis in beagle dogs after 3 years of daily oral bisphosphonate treatment. J Oral Maxillofac Surg 66: 987-994, 2008.

12. Walter C, Al-Nawas B, Grötz KA, Thomas C, Thüroff JW, Zinser V, Gamm H, Beck J and Wagner W: Prevalence and risk factors of bisphosphonate-associated osteonecrosis of the jaw in prostate cancer patients with advanced disease treated with zoledronate. Eur Urol 54: 1066-1072, 2008.

13. Reid IR, Bolland MJ and Grey AB: Is bisphosphonate-associated osteonecrosis of the jaw caused by soft tissue toxicity? Bone 41: 318-320, 2007.

14. Walter C, Pabst A, Ziebart T, Klein M and Al-Nawas B: Bisphosphonates affect migration ability and cell viability of HUVEC, fibroblasts and osteoblasts in vitro. Oral Dis 17: 194-199, 2011.

15. Giraudo E, Inoue M and Hanahan D: An amino-bisphosphonate targets MMP-9-expressing macrophages and angiogenesis to impair cervical carcinogenesis. J Clin Invest 114: 623-633, 2004.

16. Santini D, Vincenzi B, Dicuonzo G, Avvisati G, Massacesi C, Battistoni F, Gavasci M, Rocci L, Tirindelli MC, Altomare V, et al: Zoledronic acid induces significant and long-lasting modifications of circulating angiogenic factors in cancer patients. Clin Cancer Res 9: 2893-2897, 2003.

17. Cetinkaya BO, Keles GC, Ayas B and Gurgor P: Effects of risedronate on alveolar bone loss and angiogenesis: A stereologic study in rats. J Periodontol 79: 1950-1961, 2008.

18. Khandelwal VK, Mitrofan LM, Hyttinen JM, Chaudhari KR, Buccione R, Kaarniranta K, Ravingerová T and Mönkkönen J: Oxidative stress plays an important role in zoledronic acid-induced autophagy. Physiol Res 63 (Suppl 4): S601-S612, 2014.

19. Mizushima $N$ and Levine B: Autophagy in mammalian development and differentiation. Nat Cell Biol 12: 823-830, 2010.

20. Rubinsztein DC, Mariño G and Kroemer G: Autophagy and aging. Cell 146: 682-695, 2011

21. Wang IT, Chou SC and Lin YC: Zoledronic acid induces apoptosis and autophagy in cervical cancer cells. Tumour Biol 35: 11913-11920, 2014.

22. Ge XY, Yang LQ, Jiang Y, Yang WW, Fu J and Li SL: Reactive oxygen species and autophagy associated apoptosis and limitation of clonogenic survival induced by zoledronic acid in salivary adenoid cystic carcinoma cell line SACC-83. PloS One 9: e101207, 2014.

23. Lin JF, Lin YC, Lin YH, Tsai TF, Chou KY, Chen HE and Hwang TI: Zoledronic acid induces autophagic cell death in human prostate cancer cells. J Urol 185: 1490-1496, 2011.

24. Lv XH, Zhao DH, Cai SZ, Luo SY, You T, Xu BL and Chen K: Autophagy plays a protective role in cell death of osteoblasts exposure to lead chloride. Toxicol Lett 239: 131-140, 2015.

25. Bezzi M, Hasmim M, Bieler G, Dormond O and Rüegg C: Zoledronate sensitizes endothelial cells to tumor necrosis factor-induced programmed cell death: Evidence for the suppression of sustained activation of focal adhesion kinase and protein kinase B/Akt. J Biol Chem 278: 43603-43614, 2003.

26. Livak KJ and Schmittgen TD: Analysis of relative gene expression data using real-time quantitative PCR and the 2(-Delta Delta C(T)) Method. Methods 25: 402-408, 2001.

27. Klionsky DJ, Abeliovich H, Agostinis P, Agrawal DK, Aliev G, Askew DS, Baba M, Baehrecke EH, Bahr BA, Ballabio A, et al: Guidelines for the use and interpretation of assays for monitoring autophagy in higher eukaryotes. Autophagy 4: 151-175, 2008.

28. Ichimura $\mathrm{Y}$ and Komatsu M: Selective degradation of $\mathrm{p} 62$ by autophagy. Semin Immunopathol 32: 431-436, 2010.

29. Pattingre S, Espert L, Biard-Piechaczyk M and Codogno P: Regulation of macroautophagy by mTOR and Beclin 1 complexes. Biochimie 90: 313-323, 2008.

30. Esteban-Martinez L and Boya P: Autophagic flux determination in vivo and ex vivo. Methods 75: 79-86, 2015. 
31. Leist $\mathbf{M}$ and Jäättelä $\mathrm{M}$ : Four deaths and a funeral: From caspases to alternative mechanisms. Nat Rev Mol Cell Biol 2: 589-598, 2001.

32. Moscat J and Diaz-Meco MT: p62 at the crossroads of autophagy, apoptosis, and cancer. Cell 137: 1001-1004, 2009.

33. Shvets E, Fass E and Elazar Z: Utilizing flow cytometry to monitor autophagy in living mammalian cells. Autophagy 4: 621-628, 2008

34. Wasko BM, Dudakovic A and Hohl RJ: Bisphosphonates induce autophagy by depleting geranylgeranyl diphosphate. J Pharmacol Exp Ther 337: 540-546, 2011.

35. Iwai-Kanai E, Yuan H, Huang C, Sayen MR, Perry-Garza CN, Kim L and Gottlieb RA: A method to measure cardiac autophagic flux in vivo. Autophagy 4: 322-329, 2008

36. Lacy MQ, Dispenzieri A, Gertz MA, Greipp PR, Gollbach KL Hayman SR, Kumar S, Lust JA, Rajkumar SV, Russell SJ, et al: Mayo clinic consensus statement for the use of bisphosphonates in multiple myeloma. Mayo Clin Proc 81: 1047-1053, 2006.
37. Wang EP, Kaban LB, Strewler GJ, Raje N and Troulis MJ: Incidence of osteonecrosis of the jaw in patients with multiple myeloma and breast or prostate cancer on intravenous bisphosphonate therapy. J Oral Maxillofac Surg 65: 1328-1331, 2007. 38. Fournier P, Boissier S, Filleur S, Guglielmi J, Cabon F, Colombel $\mathrm{M}$ and Clézardin P: Bisphosphonates inhibit angiogenesis in vitro and testosterone-stimulated vascular regrowth in the ventral prostate in castrated rats. Cancer Res 62: 6538-6544, 2002.

39. Zhuang W, Qin Z and Liang Z: The role of autophagy in sensitizing malignant glioma cells to radiation therapy. Acta Biochim Biophys Sin (Shanghai) 41: 341-351, 2009.

40. Liang XH, Jackson S, Seaman M, Brown K, Kempkes B Hibshoosh $\mathrm{H}$ and Levine B: Induction of autophagy and inhibition of tumorigenesis by beclin 1. Nature 402: 672-676, 1999. 